\title{
Kawasaki disease in children: Epidemiology, clinical symptoms and diagnostics of 231 cases in 10 years
}

\author{
HUA ZHU, SHAO-FEI YU, YU-XIN BAI, YAN-YAN LIANG, XUE-WEN SU and JING-YING PAN \\ Department of Pediatrics, People's Hospital of Inner Mongolia, Hohhot, \\ Inner Mongolia Autonomous Region 010010, P.R. China
}

Received August 7, 2014; Accepted April 13, 2015

DOI: $10.3892 /$ etm.2015.2487

\begin{abstract}
The present study was a retrospective analysis of the dynamic changes and clinical characteristics of 231 cases of Kawasaki disease (KD) in pediatric patients admitted to the People's Hospital of Inner Mongolia between January 2003 and December 2012. A total of $37.23 \%$ of the cases occurred in the first 5 years, compared with $62.77 \%$ in the latter 5 years. The age distribution ranged from 3 months to 10 years, with a peak age of $<1$ year. The male-to-female ratio was 2.12:1, and the reoccurrence rate was $1.3 \%$. Among the patient cohort, $7.8 \%$ were Mongolian children. The most common clinical symptom was fever (87.6\%), while perianal skin peeling was the most rare (14.1\%). With regard to the analyzed biomarkers, $90.4 \%$ of patients had abnormal platelet (PLT) counts; the next highest abnormality rates were associated with erythrocyte sedimentation rate (ESR) $(74.46 \%)$ and white blood cell (WBC) counts (59.74\%), followed by levels of C-reactive protein (CRP) $(57.58 \%)$, creatinine kinase-MB (40.26\%) and hemoglobin $(\mathrm{Hb})(38.53 \%)$. In conclusion, the present study has found that approximately two-thirds of cases of KD over a 10 -year period occurred in the latter 5 years. Changes in a number of experimental indicators, including PLT, ESR and WBC, could be used in the diagnosis of the condition and to reflect the success of the clinical treatment.
\end{abstract}

\section{Introduction}

Kawasaki disease (KD), also known as mucocutaneous lymph node syndrome, is an acute disease that predominantly affects children. The primary pathological characteristics of the condition are fever and rash due to systemic vasculitis (1-3). KD mainly affects children aged between 6 months and 4 years (4). In developed countries KD is the most common

Correspondence to: Dr Hua Zhu, Department of Pediatrics, People's Hospital of Inner Mongolia, 20 Zhaowuda Road, Hohhot, Inner Mongolia Autonomous Region 010010, P.R. China

E-mail: zhuhua9966@163.com

Key words: clinical characteristics, diagnostic analysis, Kawasaki disease cause of acquired heart disease in childhood $(5,6)$. The etiology of KD has yet to be elucidated, although it is believed to be associated with the induction of an intense inflammatory host response in genetically susceptible individuals by at least one infectious agent $(7,8)$.

An epidemiological survey conducted in California between 1995 and 1999 (9) observed an increasing trend in the incidence of KD in patients aged <5 years from 1997. The incidence of KD was highest among Asian individuals, but was not associated with temperature, precipitation rates, family size or population density (9). More recent reports have revealed changes in the clinical characteristics of KD (10-12), with a study by Kang et al (13) indicating an increase in the proportion of cases of incomplete KD. This increase, as well as a decrease in the incidence of coronary artery lesions, was attributed to the early admission and management of patients with KD. The authors suggested that a diagnostic strategy for incomplete KD should be established irrespective of the presence of coronary lesions (13). Katsumata et al (14) reported the characteristics of cervical computed tomography findings in KD: They indicated that retropharyngeal lymphadenopathy and retropharyngeal edema are relatively common features of KD on CT. The more characteristics of this disease that are identified, the more help in the diagnosis and treatment of KD.

In order to enhance the understanding of the development of this disease in our local region, we performed a retrospective study of children with KD who were admitted to the People's Hospital of Inner Mongolia (Huhhot, China) over a 10 -year period with the aim of summarizing the clinical characteristics of the condition and exploring the dynamic changes of the disease over time.

\section{Patients and methods}

Patients and diagnosis. This retrospective study was conducted in the People's Hospital of Inner Mongolia, China. The medical records of patients diagnosed with KD were reviewed, and information recorded between January 2003 and December 2012 was analyzed.

A diagnosis of KD was based on the fifth edition of the KD diagnostic criteria revised by the Japanese Kawasaki Committee in 2002, as follows (2): i) Patients with $\geq 5$ of the 6 major symptoms described in the primary clinical 


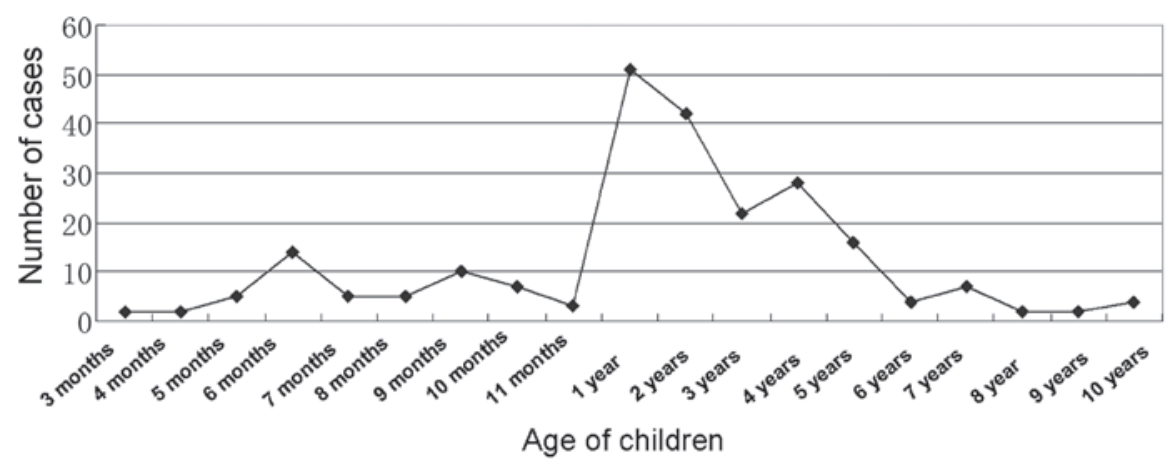

Figure 1. Age distribution of the patients with Kawasaki disease in the present study.

manifestations; ii) patients exhibiting 4 of the symptoms and either a coronary artery injury or a coronary artery aneurysm, identified during the clinical period through two-dimensional echocardiography or coronary angiography, following the exclusion of other diseases that could also be diagnosed as KD. Children meeting these diagnostic criteria, including cases transferred from other hospitals and both typical and atypical (incomplete KD in children) cases of KD, were accepted for treatment in the People's Hospital of Inner Mongolia.

The following exclusion criteria were applied: i) Patients with chronic-phase KD; ii) patients for whom a diagnosis of KD was incorrect; iii) clinical cases occurring outside the stated study period (2003-2012). The revised criteria from the Seventh World Children KD Symposium (15) were referred to for the diagnosis of coronary artery injury and aneurysms. The diagnosis of coronary artery injury was based on reference values for the internal diameter of the coronary artery: Children aged $<3$ years, $\geq 2.5 \mathrm{~mm}$; 3-9 years, $\geq 3 \mathrm{~mm}$; >9 years, $\geq 3.5 \mathrm{~mm}$. For the diagnosis of coronary aneurysm, the criteria were as follows: Mild coronary dilatation, internal diameter $\leq 4 \mathrm{~mm}$; medium coronary aneurysm, internal diameter 4-8 mm; large coronary aneurysm, internal diameter $>8 \mathrm{~mm}$.

Clinical and laboratory parameters. The clinical data for 246 cases with KD were retrospectively analyzed, and 231 cases were ultimately selected based on the aforementioned diagnostic, inclusion and exclusion criteria. The clinical characteristics of the enrolled patients, including physiological state, treatments, clinical manifestations and results of the laboratory examination and echocardiography (coronary artery and other abnormalities), were analyzed. The dynamic changes in KD were thus observed over the 10-year period.

Statistical analysis. A database containing the clinical data was established using Excel software (Microsoft Corp., Redmond, WA, USA), and statistical analyses were conducted using SPSS 13.0 software (SPSS, Inc., Chicago, IL, USA). Data are presented as the mean \pm standard deviation. Data with a normal distribution were analyzed with a variance test, while non-normally distributed data were analyzed with a rank-sum test. Count data were analyzed with a one-sided $\chi^{2}$ test. $\mathrm{P}<0.05$ was considered to indicate a statistically significant difference.

\section{Results}

Demographic data of recruited patients. A total of $37.23 \%$ $(86 / 231)$ of the cases of KD were identified in the first five years; however, this rate was significantly increased to $62.77 \%(145 / 231)$ in the last five years. The patients were aged between 3 months and 10 years, and the age of peak incidence was shown to be $<1$ year, which accounted for $23.5 \%$ of the cases. Notably, children $<5$ years old accounted for $92.5 \%$ of the total cases. The distribution of ages is shown in Fig. 1.

With regard to the gender distribution, there were 157 cases in male patients and 74 cases in female patients. The male-to-female ratio was 2.12:1, and a high risk of disease was persistently found for male patients across the 10 years.

A total of 20 patients were identified as being from an ethnic minority; among these patients, 18 were Mongolian (7.8\%) and 2 belonged to the Manchu ethnic minority $(0.9 \%)$. No higher risk of incidence was found for the Mongolian patients than for patients of other ethnicities. In addition, it was found that there was no obvious tendency of familial inheritance and seasonal preference in the incidence of KD.

Diagnosis. For the initial visit of the patients with KD, the hospital stay varied between 1 and 30 days, with an average time of 8 days and peak time of 4-7 days, which accounted for $48.3 \%$ of the cases. Among the 231 cases, 179 children (77.5\%) were hospitalized 10 days after the onset of symptoms. The majority of cases were confirmed as KD 7 days after the initial hospitalization, while a few cases were confirmed after 14 days. A total of 3 cases relapsed at their secondary treatment, and the recurrence rate was $1.3 \%$. All relapses were the first to be experienced by the patients, and the recurrence interval varied between 2 and 5 months. Among the 3 patients, 2 were male and 1 was female, and the age of recurrence was $<2$ years.

Clinical characteristics. Fever was the most common clinical characteristic and was observed in $87.6 \%$ of cases, lasting between 1 and 32 days (average, 8.47 days). Among the other 5 observed characteristics, the most frequently noted were lip changes and swollen neck glands $(65.9 \%)$, and the rarest was perianal peeling (11.4\%). Two cases exhibited swelling at the site of the Bacillus Calmette-Guérin (BCG) vaccination 
Table I. Clinical data for children with Kawasaki disease in different years.

\begin{tabular}{|c|c|c|c|c|c|c|c|c|c|c|c|c|}
\hline Clinical manifestations & $\begin{array}{c}2003 \\
(n=10)\end{array}$ & $\begin{array}{c}2004 \\
(n=21)\end{array}$ & $\begin{array}{c}2005 \\
(n=24)\end{array}$ & $\begin{array}{c}2006 \\
(n=10)\end{array}$ & $\begin{array}{c}2007 \\
(n=19)\end{array}$ & $\begin{array}{c}2008 \\
(n=23)\end{array}$ & $\begin{array}{c}2009 \\
(n=26)\end{array}$ & $\begin{array}{c}2010 \\
(n=37)\end{array}$ & $\begin{array}{c}2011 \\
(n=26)\end{array}$ & $\begin{array}{c}2012 \\
(n=35)\end{array}$ & $\chi^{2}$ & P-value \\
\hline Fever lasting $>5$ days & 9 & 19 & 23 & 10 & 16 & 21 & 26 & 33 & 23 & 30 & 4.98 & 0.026 \\
\hline Distal extremity changes & 9 & 10 & 10 & 7 & 10 & 9 & 16 & 26 & 23 & 21 & 5.49 & 0.780 \\
\hline Skin rash & 3 & 12 & 9 & 7 & 10 & 9 & 17 & 26 & 19 & 21 & 3.40 & 0.046 \\
\hline Conjunctival hyperemia & 9 & 14 & 14 & 9 & 12 & 10 & 14 & 28 & 19 & 24 & 4.60 & 0.032 \\
\hline Lip changes & 5 & 16 & 14 & 9 & 14 & 14 & 16 & 24 & 21 & 24 & 5.19 & 0.820 \\
\hline Cervical lymphadenopathy & 7 & 14 & 17 & 7 & 9 & 17 & 17 & 24 & 19 & 23 & 3.43 & 0.064 \\
\hline
\end{tabular}

Table II. Laboratory examination results.

\begin{tabular}{lccc}
\hline Laboratory examination & Mean \pm SD & Available cases/abnormal cases (n/n) & Abnormality rate $(\%)$ \\
\hline CRP, mg/l & $64.38 \pm 47.07$ & $216 / 133$ & 61.57 \\
ESR, mm/h & $55.8 \pm 30.51$ & $217 / 172$ & 79.26 \\
WBC count, $10^{9} / 1$ & $13.20 \pm 6.07$ & $226 / 138$ & 61.06 \\
Hb, g/l & $111.27 \pm 13.73$ & $226 / 89$ & 39.38 \\
PLT count, $10^{9} / 1$ & $378.52 \pm 158.24$ & $226 / 208$ & 92.04 \\
CK-MB, U/l & $19.44 \pm 9.92$ & $182 / 93$ & 51.10 \\
\hline
\end{tabular}

CRP, C-reactive protein; ESR, erythrocyte sedimentation rate; WBC, white blood cell; Hb, hemoglobin; PLT, platelet; CK-MB, creatinine kinase-MB; SD, standard deviation.

Table III. Echocardiography results.

\begin{tabular}{lcr}
\hline Disease & Number of cases & Rate $(\%)$ \\
\hline Left coronary artery & 38 & 16.45 \\
Right coronary artery & 12 & 5.19 \\
Bilateral coronary dilatation & 78 & 33.77 \\
Tricuspid regurgitation & 56 & 24.24 \\
Mitral regurgitation & 16 & 6.93 \\
Pericardial effusion & 21 & 9.09 \\
Myocarditis & 16 & 6.93 \\
Coronary artery aneurysms & 2 & 0.87 \\
\hline
\end{tabular}

(Table I). The differences among the occurrence of different symptoms was statistically significant, while the differences among the distribution of the symptoms across the study period were not statistically significant. Statistical analysis also showed that the incidence of atypical KD increased from $10.38 \%$ in 2003 to $40.12 \%$ in 2012 .

Among the 231 cases of KD, 59 patients (25.54\%) exhibited other complications. There were 34 cases $(14.72 \%)$ of lower respiratory tract infection with abnormal chest X-ray findings; most showed increased lung markings and one had severe pneumonia. In addition, there were 3 cases of peripheral hydrocephalus, 3 cases of urinary tract infection and 3 cases of infantile diarrhea with dehydration. A further 2 cases exhibited the complications of leukemia and aplastic anemia.
Laboratory examination results. The laboratory examination results of 231 cases are shown in Table II. For the white blood cell (WBC) count and the levels of C-reactive protein (CRP), no statistically significant differences were found between the number of normal and abnormal results. In the 226 cases with available hemoglobin $(\mathrm{Hb})$ data, the incidence of anemia was found to be higher in children aged $<2$ years; among these cases, 14 exhibited severe anemia (6.19\%). In the 226 cases with available platelet (PLT) data, 41 patients exhibited enhanced PLT counts in the course of 1-4 days $(17.75 \%)$ and 88 cases exhibited enhanced PLT counts in the course of 5-10 days (38.10\%); in total, abnormal PLT counts were found in 208 cases $(90.04 \%)$ over an extended period ( $>10$ days). As the disease attenuated, the PLT counts returned to normal levels. No statistically significant differences were found in the incidence of coronary artery injury between patients with enhanced and normal PLT levels $(\mathrm{t}=-1.001, \mathrm{P}=0.321)$ or between patients with enhanced and normal creatinine kinase-MB (CK-MB) levels $(\mathrm{t}=-1.346$, $\mathrm{P}=0.183)$.

Echocardiography. Echocardiography was performed in all 231 cases. A total of 146 patients $(63.20 \%)$ were found to have coronary artery disease; 144 of these cases were identified as coronary artery expansion and 2 were identified as coronary artery aneurysms. The results of the echocardiography are shown in Table III. Among the children with coronary artery disease, $67.12 \%(98 / 146)$ were treated in the primary hospital (such as the village, town or community hospital) for the first time. 


\section{Discussion}

Since 1993, a large number of studies (16-19) have been performed in different areas of China to investigate the epidemiology and clinic characteristics of KD, with a focus on the differences among the different regions. The present study was a retrospective study of patients with KD hospitalized in the People's Hospital of Inner Mongolia over a 10-year period. Compared with a previous report (17) on the incidence and symptoms of the condition, an overall steady increase in the incidence of KD was observed.

The results of the present study showed that approximately two-thirds of the cases of KD occurred in the latter 5 years of the 10-year period. The age distribution of the children with KD ranged between 3 months and 10 years, with a peak incidence in children aged $<1$ year. This finding was consistent with the results of previous epidemiological investigations in Shanghai $(17,18)$ and Japan $(20)$, while the age was a little younger than results from other regions, including Beijing and Jilin (1-2 years old) $(21,22)$. The gender ratio of KD was shown to be 2.12:1, which was lower than the ratios found in Zhejiang (2.3:1) (19) and Guangdong (2.23:1) (23) and higher than those in other regions of China, including Taiwan (1.62:1), and Japan (1.32:1) (20-22). The recurrence rate of KD was $1.3 \%$, which was consistent with that in reports from Beijing (1.4\%) and Shanghai $(1.2 \%)$ in the same period and lower than that reported for Japan (2.2\%) (20). The present study showed that the incidence of KD in Mongolian children was not significantly higher than that in other ethnic groups, and no significant family tendency was observed for this disease. With regard to the clinical manifestations of KD, the present results were similar to but slightly different from those of previous reports (17-19). In 2 cases, redness was found at the site of the $\mathrm{BCG}$ vaccination, which may serve as a reference for the early diagnosis of KD.

Consistent with improvements in the diagnosis and treatment of the condition, the incidence of atypical KD in the present study showed an increasing trend, which is consistent with reports from other regions of China (16-19,23).

In recent years, laboratory examinations for children with KD have shown considerable improvements. Parameters such as WBC, PLT, Hb, CRP and erythrocyte sedimentation rate (ESR) have been routinely used in the diagnosis of KD and have become essential indicators for observing the dynamic changes in the disease, despite their non-specificity, due to the wide-spread abnormalities in these parameters in the majority of patients with KD (24-26). Increases in CRP and ESR in the acute phase have been identified as diagnostic indicators for both typical and atypical KD (27). Currently, the reference values for the diagnosis of incomplete KD are as follows: CRP $\geq 30 \mathrm{mg} / \mathrm{l}$ or ESR $>40 \mathrm{~mm} / \mathrm{h}$ (28); these values should be combined with clinical observations. In the present study, $61.06 \%$ of patients were observed to have an increased WBC count in the acute phase, with the remaining patients exhibiting a delayed increase following the acute phase, which suggests a lack of support for the association between the incidence of KD and infection.

No significant association was observed between an increased PLT count and the occurrence of coronary artery injury among the 231 cases; this result varied from that of another report, in which it was concluded that an increase in

\section{Occurrence of Kawasaki disease}

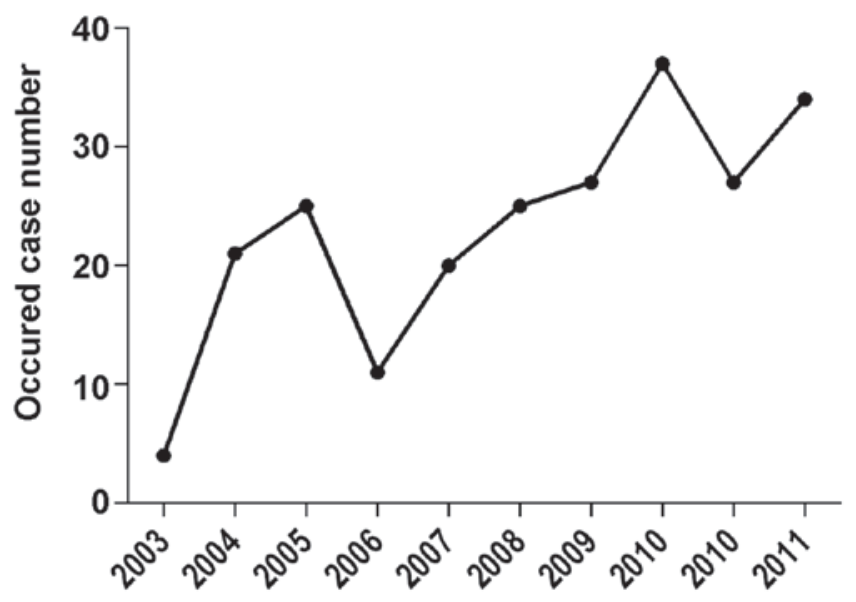

Figure 2. Occurrences of Kawasaki disease between 2003 and 2011.

PLT levels was a risk factor for coronary artery injury among KD cases (29). This difference may have been associated with the small sample size in the present study. In addition, the present study showed a lack of association between increased CK-MB and coronary artery injury, which contradicted a previous report in which the levels of CRP and CK-MB were significantly correlated in cases of KD and were suggested to be important predictors of coronary artery and myocardial damage (30). Further verification is therefore required for any definite conclusion.

In the present study, $63.20 \%$ of the total 231 patients with KD were found to have coronary artery disease, which is similar to the result from Jilin (63.26\%) during the same period, while generally higher than the results from the rest of the country and significantly higher than those from Japan (31) and South Korea (32). Notably, fever was the most common clinical observation in the primary diagnosis of patients with KD and with a risk of coronary artery disease; this was attributed to the scattered residence of patients living in Inner Mongolia, as well as the limited medical care available to these patients, leading to a delay in treatment and the aggravation of disease. The 2 patients with coronary aneurysms in this study were both from remote areas; this has not been reported in previous studies. By reviewing the dynamic changes in KD over a 10-year period, an arc-shaped change can be observed for the general trend of coronary artery injury in KD. In the first 3 years the occurrence of KD showed a steady increase (Fig. 2), which could be attributed to the lack of knowledge about atypical KD and the referral of numerous patients to more advanced hospitals in the adjacent provinces; in the middle 4 years, with the improvement of clinical conditions, the rates of KD detection increased; in the latter 3 years, the early diagnosis and treatment of KD effectively prevented the occurrence of coronary artery injury, particularly in the final two years when the majority of the cases of coronary artery disease were found to be mild expansions, with few cases of severe coronary artery aneurysms and expansion. At present, there are few reports concerning the dynamics changes in coronary artery injury in KD in the country $(33,34)$. 
In conclusion, KD has become a major cause of acquired heart disease in children $(2,35)$. Considering the threat of coronary artery injury to the health of the patient, it is suggested that clinical staff, as well as researchers, should pay more attention to the early diagnosis and standard treatment of KD, as well as long-term follow-up visits. Furthermore, it is necessary to enhance the clinical knowledge of KD in primary hospitals, such as village hospitals.

\section{References}

1. Ogata S, Tremoulet AH, Sato Y, et al: Coronary artery outcomes among children with Kawasaki disease in the United States and Japan. Int J Cardiol 168: 3825-3828, 2013.

2. Ayusawa M, Sonobe T,Uemura S, et al; Kawasaki Disease Research Committee; Revision of diagnostic guidelines for Kawasaki disease (the 5th revised edition). Pediatr Int 47: 232-234, 2005.

3. Newburger JW, Takahashi M, Gerber MA, et al; Committee on Rheumatic Fever, Endocarditis, and Kawasaki Disease; Council on Cardiovascular Disease in the Young; American Heart Association: Diagnosis, treatment, and long-term management of Kawasaki disease: A statement for health professionals from the Committee on Rheumatic Fever, Endocarditis, and Kawasak Disease, Council on Cardiovascular Disease in the Young, American Heart Association. Pediatrics 114: 1708-1733, 2004.

4. Bayers S, Shulman ST and Paller AS: Kawasaki disease: Part I. Diagnosis, clinical features, and pathogenesis. J Am Acad Dermatol 69: 501, 2013.

5. JCS Joint Working Group: Guidelines for diagnosis and management of cardiovascular sequelae in Kawasaki disease (JCS 2008) - digest version. Circ J 74: 1989-2020, 2010.

6. Eleftheriou D, Levin M, Shingadia D, Tulloh R, Klein NJ and Brogan PA: Management of Kawasaki disease. Arch Dis Child 99: 74-83, 2014.

7. Emi M, Keicho N, Tokunaga K, et al: Association of diffuse panbronchiolitis with microsatellite polymorphism of the human interleukin 8 (IL-8) gene. J Hum Genet 44: 169-172, 1999.

8. Kaneko K, Obinata K, Katsumata K, Tawa T, Hosaka A and Yamashiro Y: Kawasaki disease in a father and daughter. Acta Paediatr 88: 791-792, 1999.

9. Chang RK: Epidemiologic characteristics of children hospitalized for Kawasaki disease in California. Pediatr Infect Dis J 21: 1150-1155, 2002.

10. Golshevsky D, Cheung M and Burgner D: Kawasaki disease - the importance of prompt recognition and early referral. Aust Fam Physician 42: 473-476, 2013.

11. Daniels LB, Gordon JB and Burns JC: Kawasaki disease: Late cardiovascular sequelae. Curr Opin Cardiol 27: 572-577, 2012.

12. Neudorf U: Kawasaki disease in children and adolescents. Z Rheumatol 70: 838-843, 2011 (In German).

13. Kang HJ, Kim GN and Kil HR: Changes of clinical characteristics and outcomes in patients with Kawasaki disease over the past 7 years in a single center study. Korean J Pediatr 56: 389-395, 2013.

14. Katsumata N, Aoki J, Tashiro M, Taketomi-Takahashi A and Tsushima Y: Characteristics of cervical computed tomography findings in Kawasaki disease: A single-center experience. J Comput Assist Tomogr 37: 681-685, 2013.

15. JCS Joint Working Group: Guidelines for diagnosis and management of cardiovascular sequelae in Kawasaki disease (JCS 2013). Digest version. Circ J 78: 2521-2562, 2014.
16. Jiao F, Yang L, Qiao J, Li Y, Zhang T and Liu C: Epidemiological and clinical characteristics of Kawasaki disease in Shanxi province. Zhonghua Liu Xing Bing Xue Za Zhi 21: 97-99, 2000 (In Chinese)

17. Jiao F, Yang L, Li Y, et al: Epidemiologic and clinical characteristics of Kawasaki disease in Shaanxi Province, China, 1993-1997. J Trop Pediatr 47: 54-56, 2001.

18. Wang D, Hu B, Wang F, Zhang T and Liu C: Study on the epidemiological features of Kawasaki disease in Jiangsu. Zhonghua Liu Xing Bing Xue Za Zhi 21: 94-96, 2000 (In Chinese).

19. Zou LX and Gong FQ: Clinical features of recurrent Kawasaki disease in 20 children. Zhongguo Dang Dai Er Ke Za Zhi 10: 617-619, 2008 (In Chinese).

20. Nakamura Y, Yashiro M, Uehara R, et al: Epidemiologic features of Kawasaki disease in Japan: Results of the 2009-2010 nationwide survey. Tainjin Yi Yao 22: 216-221, 2012.

21. Kayiran SM, Dindar A and Gurakan B: An evaluation of children with Kawasaki disease in Istanbul: A retrospective follow-up study. Shiyong Yi Xue Za Zhi (Sao Paulo) 65: 1261-1265, 2010.

22. Zhang X, Zhang Z, Liu S and Sun J: Epidemiologic survey of Kawasaki disease in Jilin from 1999 through 2008. Pediatr Cardiol 33: 272-279, 2012

23. Li XH, Li XJ, Li H, Xu M and Zhou M: Epidemiological survey of Kawasaki disease in Sichuan province of China. J Trop Pediatr 54: 133-136, 2008.

24. Satou GM, Giamelli J and Gewitz MH: Kawasaki disease: diagnosis, management, and long-term implications. Cardiol Rev 15: 163-169, 2007.

25. Yi DY, Kim JY, Choi EY, Choi JY and Yang HR: Hepatobiliary risk factors for clinical outcome of Kawasaki disease in children. BMC Pediatr 14: 51, 2014.

26. Schnautz LS and Leggett P: Kawasaki disease: A ride for little girls too! Crit Care Nurs Clin North Am 20: 265-271, 2008.

27. Liu R, He B, Gao F, Liu Q and Yi Q: Relationship between adipokines and coronary artery aneurysm in children with Kawasaki disease. Transl Res 160: 131-136, 2012.

28. Billoo AG, Lone SW, Siddiqui S and Atiq H: Incomplete Kawaski disease: Are we missing it? J Pak Med Assoc 59: 42-43, 2009

29. Fu J, Zhang HY and Yang YZ: High risk factors of coronary artery lesions caused by Kawasaki Disease. Tianjin Med J 40: 679-681, 2012 (In Chinese).

30. Chen QJ, Lei XM, Zuo YX and Jiang L: Relationship between serum C-reaction protein and myocardiac enzymology, coronary artery lesions in children with Kawasaki Disease. J Pract Med 22: 4116-4117, 2010 (In Chinese)

31. Ogawa S: Biochemical and immunological laboratory findings in Kawasaki disease. Nihon Rinsho 66: 315-320, 2008 (In Japanese).

32. Park YW, Han JW, Hong YM, et al: Epidemiological features of Kawasaki disease in Korea, 2006-2008. Pediatr Int 53: 36-39, 2011.

33. Xing Y, Wang H, Yu X, Chen R and Hou Y: Assessment of coronary artery lesions in children with Kawasaki disease: Evaluation of MSCT in comparison with 2-D echocardiography. Pediatr Radiol 39: 1209-1215, 2009.

34. Xu MG, Men LN, Zhao CY, et al: The number and function of circulating endothelial progenitor cells in patients with Kawasaki disease. Eur J Pediatr 169: 289-296, 2010.

35. Dimitriades VR, Brown AG and Gedalia A: Kawasaki disease: Pathophysiology, clinical manifestations, and management. Curr Rheumatol Rep 16: 423, 2014. 